Orthopäde 2009 · 38:883-884

DOI 10.1007/s00132-009-1478-7

Online publiziert: 2. September 2009

(c) Springer Medizin Verlag 2009

\author{
L. Weh \\ Rückenzentrum am Markgrafenpark, Berlin
}

\title{
Wir müssen die Versorgung chronisch Rückenkranker grundsätzlich ändern!
}

tionsindikationen $\mathrm{zu}$ weit gestellt. Fatal ist, dass nicht wirksame Therapieformen symptomunterhaltend sind, die Chronifizierungsrate weiter steigern und das Heer der Rückenkranken vermehren. Ein Bärendienst an der Bevölkerung. Im internationalen Vergleich schneiden wir Deutschen bei der beruflichen Wiedereingliederung (zusammen mit den Dänen) am schlechtesten ab. Die „Returnto-work-Raten “ bei rückenschmerzbedingter 12-wöchiger Arbeitsunfähigkeit ist deprimierend niedrig [2]. Bei uns nehmen nur $54 \%$ in den folgenden 2 Jahren ihre Arbeit wieder auf - in den Niederlanden sind es $81 \%$. Der „Sachverständigenrat für die Konzertierte Aktion im Gesundheitswesen“ spricht bei Patienten mit Rückenleiden von einer „Über-, Unter- und Fehlversorgung“. Demnach hätten wir eine Überversorgung bei der operativen Therapie, der bildgebenden Diagnostik, bei Injektionen und der Verordnung von Bettruhe sowie bei der passiven physikalischen Therapie. Unterversorgt hingegen seien sie bei der Beratung und psychosozialen Unterstützung. Der Rat empfiehlt, die Rückenschmerzbehandlung auf der Grundlage evidenzbasierter Leitlinien zu optimieren. Diese Analyse trifft den Nagel auf den Kopf und wird dennoch geflissentlich ignoriert - nicht ganz folgenlos: immerhin haben wir die mittlerweile teuerste Volkskrankheit produziert!

Tausendfach werden täglich wissenschaftlich unsichere Maßnahmen in der täglichen Praxis geleistet - der Großteil der Infiltrationen, Akupunkturen, Elektrotherapie und Krankengymnastik gehört dazu. Tausendfach werden Opera-

\section{( Patienten mit Rückenleiden: „Über-, unter- und fehlverorgt."}

Es muss die Frage erlaubt sein, inwieweit unsere chronische Nichtbeachtung der Datenlage zur Therapieeffizienz eine Mitschuld am eklatanten Anstieg der Rückenschmerzpatienten trägt. Oder anders gesagt: Es kann nicht sein, dass wir wider besseres Wissen weitermachen wie bisher.

Bei chronischen Rückenschmerzen ist die interdisziplinäre multimodale Therapie „Goldstandard“. Tückischerweise vollzieht sich der Übergang von der akuten in die chronische Phase fast unmerklich. Der Bandscheibenvorfall mit handfestem morphologischem Befund wandelt sich undramatisch im Laufe der Monate zum biopsychosozialen Phänomen. Der ungeschulte Beobachter mag den kleinen aber essentiellen Wandel übersehen: Keine sinnfällige Zäsur drängt während der
Chronifizierungsphase zur Abkehr von der monomodalen Therapie hin zum Bearbeiten der „yellow flags“! Im Gegenteil, die Patienten lassen sich nur ungern von ihren bisherigen Bewegungs- oder Essgewohnheiten abbringen. Psychosoziale Aspekte sind oft gerade in betroffenen Köpfen tabuisiert. Passive Therapieformen sind beliebt. Die konfliktfreie Fortführung der Therapie „as usual“ wird einem leicht gemacht.

Definitionen und Grundsätze der interdisziplinären Therapie wurde kürzlich von der Ad-hoc-Kommission der DGSS zusammengefasst und werden in diesem Heft zitiert [1]. Eine diesbezügliche ärztliche Bewusstseinsbildung und Anpassung des Praxisalltags an die eindeutigen wissenschaftlichen Erkenntnisse sind notwendig. Wir benötigen Rahmenbedingungen, welche die interdisziplinäre Therapie in der täglichen Praxis weiter etablieren helfen. Die Schaffung weiterer interdisziplinärer Verbünde im kassenärztlichen Bereich ist dringend $\mathrm{zu}$ fördern.

Im vorliegenden Themenheft „Nichtoperative Rückenschmerzbehandlung“ wird v. a. die chronische Problematik thematisiert. Als Autoren gewonnen werden konnten Wegbereiter und Träger der konservativen Versorgung. Im Hinblick auf die Unverzichtbarkeit der interdisziplinären Therapie beim chronischen Rückenschmerz werden psychologische, physio- und sporttherapeutische Arbeiten präsentiert. Bei Letzteren wollen wir auch die nötige intelligente Steuerung des Belastungsaufbaus unter permanenter Berücksichtigung psychologischer Aspekte 
darstellen. Dies geht konzeptionell weit über die bisher geübte Trainingstherapie hinaus. Die psychosoziale Betreuung ist im Team so wichtig wie vielfältig. Sie ermöglicht und begleitet die Physiotherapie durch Abbau der Angst („fear avoidance behaviour"), definiert Ziele (z. B. den beruflichen Wiedereinstieg) und hilft bei deren Erlangung (z. B. durch Sozialberatung). Hierbei sind Orthopäden alleine schlichtweg überfordert - und die positiven Ergebnisse der interdisziplinären multimodalen Therapie sprechen für sich!

Wir hoffen die Essentials einer modernen und effektiven interdisziplinären Behandlung des Rückenschmerzes in diesem Themenheft darstellen zu können.
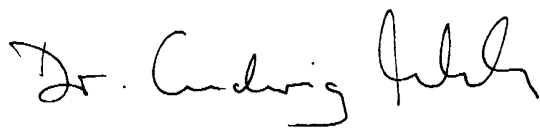

L. Weh

\section{Korrespondenzadresse \\ Dr. L.Weh}

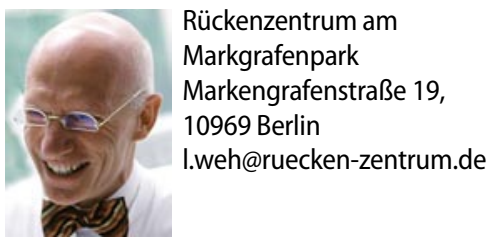

\section{Literatur}

1. Arnold B, Brinkschmidt T, Casser HR et al (2009) Multimodale Schmerztherapie, Konzepte und Indikation. Schmerz 23:112-120

2. Bloch S, Prins R (2001) Who returns to work and why? A six country study on work incapacity and reintegration. Transaction Publisher, New Brunswick

\section{Der Unfallchirurg}

Die Zeitschrift „Der Unfallchirurg“ bietet Ihnen jeden Monat umfassende und aktuelle Beiträge zu interessanten Themen aus allen Bereichen der Unfall- und Wiederherstellungschirurgie.

\section{Heft 10/2009:}

- Standardisierte Primärtherapie des polytraumatisierten Patienten

- Chirurgisches Management vital bedrohlicher Verletzungen

- Verteilungsplanung von Verletzten beim MANV oder Katastrophenfall

- Damage Control Orthopedics. Was ist der aktuelle Stand?

- CME: Der derzeitige Stand der minimal invasiven Plattenosteosynthese

\section{Heft 11/2009:}

- Prophylaktische Beatmung des Schwerverletzten mit Thoraxtrauma

- Gerinnungsmanagement beim Polytrauma

- Bedeutung der Hypothermie beim Polytrauma

- Wirtschaftliche Herausforderungen der Polytraumaversorgung

- CME: Aktueller Stand der minimal invasiven Plattenosteosynthese. Teil 2: Spezielles

\section{Der Unfallchirurg + Der Orthopäde:}

Jetzt zum Kombipreis abonnieren und bis zu 219 Euro sparen!

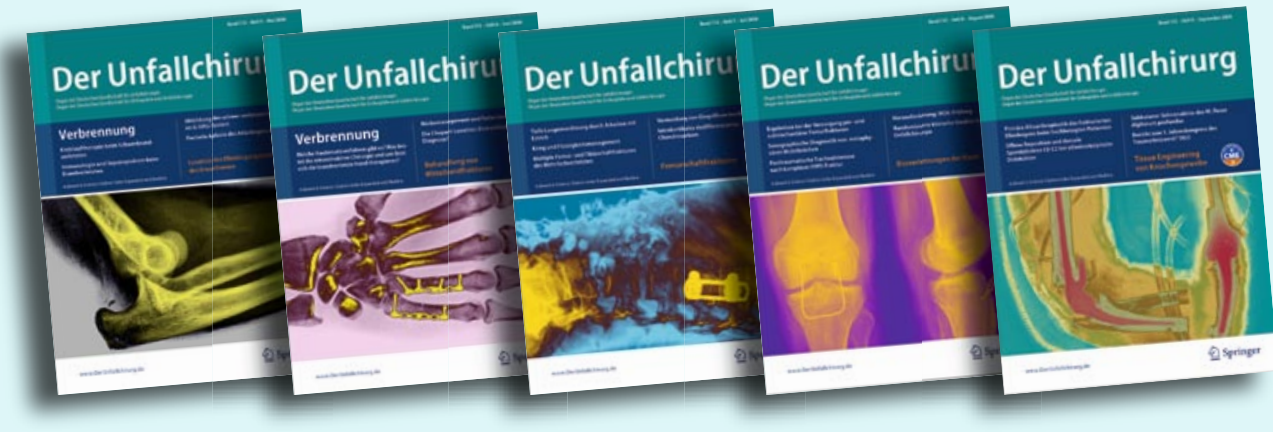

24 Hefte jährlich für 399,- EUR. Ärztinnen/Ärzte in Aus- und Weiterbildung erhalten das Kombiabo zum Vorzugspreis von 239,40 EUR. Alle Preise inkl. 7\% MwSt, zzgl. 39 EUR Versandkosten Inland.

Weitere Informationen und Bestellmöglichkeit direkt beim Springer Kundenservice: Springer Customer Service Center

Kundenservice Zeitschriften

Haberstr. 7

69126 Heidelberg

Tel. +49/6221/345-4303; Fax: -4229

leserservice@springer.com

www. DerUnfallchirurg.de 